\title{
Using mHealth to Improve Usage of Antenatal Care, Postnatal Care, and Immunization: A Systematic Review of the Literature
}

\author{
Jessica L. Watterson, ${ }^{1}$ Julia Walsh, ${ }^{2}$ and Isheeta Madeka ${ }^{3}$ \\ ${ }^{1}$ Private Practice in Maternal and Child Health, Berkeley, CA 94704, USA \\ ${ }^{2} 207 L$ University Hall, University of California, Berkeley, CA 94704, USA \\ ${ }^{3}$ University of California, Berkeley, CA 94704, USA \\ Correspondence should be addressed to Jessica L. Watterson; jessica.watterson@gmail.com
}

Received 21 November 2014; Revised 19 January 2015; Accepted 22 January 2015

Academic Editor: Pascale Allotey

Copyright (c) 2015 Jessica L. Watterson et al. This is an open access article distributed under the Creative Commons Attribution License, which permits unrestricted use, distribution, and reproduction in any medium, provided the original work is properly cited.

\begin{abstract}
Mobile health (mHealth) technologies have been implemented in many low- and middle-income countries to address challenges in maternal and child health. Many of these technologies attempt to influence patients', caretakers', or health workers' behavior. The purpose of this study was to conduct a systematic review of the literature to determine what evidence exists for the effectiveness of mHealth tools to increase the coverage and use of antenatal care (ANC), postnatal care (PNC), and childhood immunizations through behavior change in low- and middle-income countries. The full text of 53 articles was reviewed and 10 articles were identified that met all inclusion criteria. The majority of studies used text or voice message reminders to influence patient behavior change $(80 \%, n=8)$ and most were conducted in African countries $(80 \%, n=8)$. All studies showed at least some evidence of effectiveness at changing behavior to improve antenatal care attendance, postnatal care attendance, or childhood immunization rates. However, many of the studies were observational and further rigorous evaluation of mHealth programs is needed in a broader variety of settings.
\end{abstract}

\section{Introduction}

Despite ongoing efforts to improve maternal and child health in developing countries, mortality rates remain much higher than in developed countries. Women in developing regions face a lifetime risk of maternal death of 1 in 160, as compared with 1 in 3700 for women living in developed regions [1]. These inequalities are driven by many causes, one of which is limited access to preventive services. For example, in lowand middle-income countries, only about $52 \%$ of pregnant women receive the World Health Organization- (WHO-) recommended minimum of four antenatal visits [2]. The postnatal period is also critical to the health of a mother and newborn, as the majority of postnatal maternal deaths happen during the first week after birth [3]. However, a recent analysis of Demographic and Health Surveys for 23 African countries found that, of the two-thirds of women giving birth at home, only $13 \%$ received a postnatal check-up within two days [3]. Immunization is another critical preventive service that can save the lives of many infants and children. Despite being one of the most cost-effective tools for saving lives, nearly one in five children globally did not receive their full package of immunizations in 2012 and 1.5 million children under the age of 5 died from vaccine-preventable diseases in $2008[4,5]$. Antenatal care (ANC), postnatal care (PNC), and childhood immunization make up an important package of preventive services that can improve maternal and child health. Families tend to use medical services when someone is ill but frequently omit these beneficial preventive services that are essential to improve health.

The field of mHealth, or mobile health, has been proposed as a potential solution to many of the challenges that developing countries face, including workforce shortages, lack of health information, limited training for health workers, and difficulty tracking patients. mHealth projects have been implemented all over the world, using mobile phones for 
record keeping, data collection, or patient communication [6]. Further, mHealth tools have been used to promote behavior change in health workers and/or patients. For example, text message reminders have been shown to increase careseeking behavior or medication adherence in some patients and mobile data collection and communication tools for health workers have improved follow-up of patients and data reporting [7-9].

Though there are relatively few thorough evaluations of mHealth programs [6], some published studies do exist. Given that mHealth tools have shown some promise for behavior change more broadly, there is potential for this field to improve essential preventive maternal and child health services as well. Based on the existing evidence in peer-reviewed publications, this literature review aims to determine the effectiveness of mHealth tools to increase the coverage and use of antenatal care, postnatal care, and childhood immunizations through behavior change in lowand middle-income countries.

\section{Materials and Methods}

2.1. Information Sources. This literature review was conducted through a keyword search of the following databases to identify relevant peer-reviewed articles: Google Scholar, PubMed, Embase, PsycINFO, and EBSCO Host. Keywords used in these searches included mHealth, mobile health, mobile phone, reminder, recall, mobile medical records, antenatal care, postnatal care, and immunization.

2.2. Inclusion Criteria. In order to be included in the review, the article had to meet the following inclusion criteria:

(i) study was evaluating an mHealth intervention targeted at increasing antenatal care attendance, postnatal care attendance, or childhood immunization rates through behavior change;

(ii) study was implemented in a low- or middle-income country;

(iii) study included measurement of process, behavior change, health, or quality of care outcomes (i.e., studies were excluded that only evaluated willingness of participants to receive an mHealth intervention, without implementing it);

(iv) study was a peer-reviewed article;

(v) study was available in English;

(vi) study was published between January 1, 2000 and November 20, 2014.

These criteria were selected to ensure that the included studies examined outcomes of existing mHealth interventions, not exploratory studies or protocols that have not been implemented yet. Low-, middle-, or high-income status for countries was determined using the World Bank's 2014 classification, which is based on estimates of the gross national income per capita for the previous year [10]. In addition, the inclusion of only peer-reviewed articles helped to ensure that higher quality studies were examined. Though there have been well-designed studies using mHealth for behavior change to support maternal and child health in high-income countries, these studies were excluded due to the resource disparities between high-income countries and others. Issues with prevalence of mobile phones and consistency of power and internet access are shared across many low- and middleincome countries and therefore these studies are more comparable than those conducted in high-income countries. No keywords for "low- or middle-income countries" were used in the searches, as these keywords might have excluded relevant results if the study was not specifically labeled as such. Instead, the authors screened manually for this criterion. The review was limited to studies available in English, though this is a limitation of this review, and future reviews should include additional languages, if feasible. Finally, studies only included those that were published after 2000, as mobile technologies were not widely available, especially in low- and middle-income countries, prior to that time.

2.3. Study Selection and Data Collection. The database searches were undertaken by two researchers (Jessica L. Watterson and Isheeta Madeka) between November 10, 2014 and January 18, 2015. Subsequent review of results was undertaken by one researcher (Jessica L. Watterson). The resulting articles were first screened by title, then by abstract, and finally by full text to progressively eliminate articles not meeting the inclusion criteria. Many systematic reviews of mHealth research were identified in the results $(n=26)$, so the included articles and reference lists of these reviews were all examined to ensure an exhaustive search. Finally, the references of all included articles were reviewed as well.

The results of study screening and selection are illustrated in Figure 1. The database searches identified 1,899 articles initially. After removing duplicates, 508 records remained. Each of these records was screened by title and abstract (if necessary), and 455 records were excluded after this preliminary review. The full text of the remaining 53 articles was reviewed to determine if they met the inclusion criteria. 43 of the articles were excluded and the reasons for exclusion included study being conducted in a high-income country $(n=5)$; not studying antenatal care attendance, postnatal care attendance, or childhood immunization rates $(n=7)$; not studying an mHealth intervention $(n=2)$; or only providing program descriptions or a protocol but no evaluation data $(n=4)$. The other 25 articles that were excluded were mHealth literature reviews that did not identify any new articles for review. One article outlined a protocol for a study that will be very relevant once complete; however it was nevertheless excluded because no evaluation data is published yet [11].

2.4. Quality Assessment. Risk of bias was assessed for all included randomized controlled trials (RCTs) $(n=2)$ using the Cochrane Risk of Bias Assessment Tool [12]. This tool was introduced in 2008 by the Cochrane Collaboration and can be used to assess risk of bias in a study by evaluating a study's allocation sequence generation (randomization), 

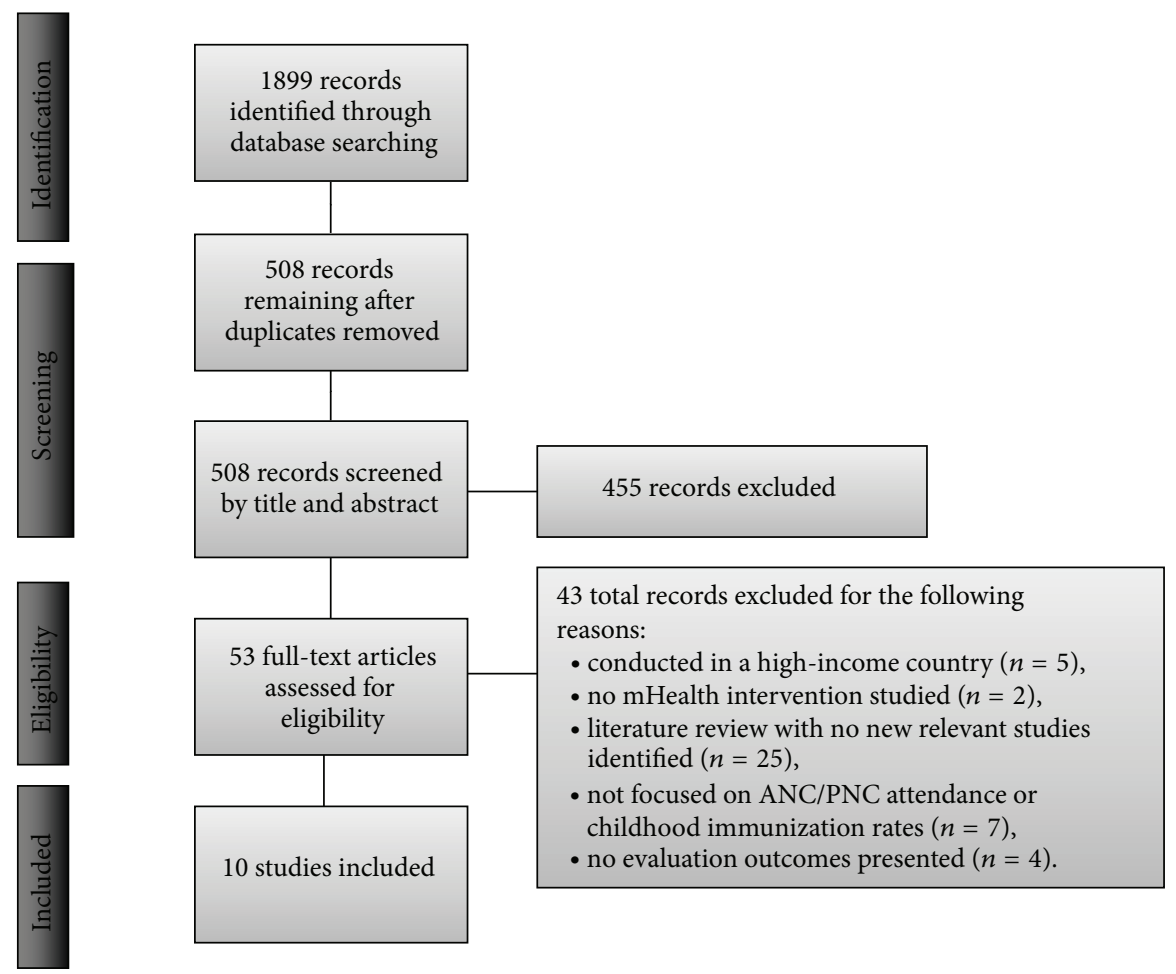

Figure 1: PRISMA flow diagram [26].

allocation concealment, blinding, incomplete data, selective reporting, and other potential threats to the study's validity. The quality of the observational studies $(n=8)$ was assessed using the Newcastle-Ottawa Quality Assessment Scale [13]. This tool was developed by the universities of Newcastle, Australia, and Ottawa, Canada, and assessed the quality of nonrandomized studies by evaluating potential sources of bias in the selection and comparability of participants, the assessment of outcomes, and the duration and adequacy of follow-up. Scores are awarded out of 9 possible points, with higher scores indicating higher study quality.

2.5. Synthesis of Results. The primary author (Jessica L. Watterson) extracted information from included articles for tabulation in an Excel spreadsheet. The information extracted included type of study, summary conclusions, methods used, intervention studied, health issue(s) studied, outcomes measured, sample size, intervention frequency, effectiveness of intervention, study quality, study location, clinical characteristics/setting, mHealth tools used, and project name (if any).

\section{Results}

Most articles examined process and behavior change outcomes and made recommendations for future mHealth programs and suggested further research. The study characteristics and key outcomes for each included article are outlined in Table 1.
3.1. Characteristics of Studies. In total, ten articles satisfied the inclusion criteria. Of these, two studies were RCTs $[14,15]$ and the other eight were observational studies [16-23]. Four of the observational studies attempted to limit sources of bias (though not as rigorously as the RCTs) by using a historic control group [16,17] or nonrandomized control group [19] or measuring outcomes before and after implementation of the mHealth intervention [18]. The remaining four observational studies did not use a control group [18-21], and as such the outcomes of these studies are less reliable.

Seven $(70 \%)$ of the articles studied antenatal care attendance $[14,15,17-20,22]$; two (20\%) studied postnatal care attendance $[16,20]$; and four (40\%) studied childhood immunization rates $[18,20,21,23]$. Eight $(80 \%)$ of the studies used an mHealth intervention that sent reminders to seek care directly to patients $[14-18,20,21,23]$ and five (50\%) sent educational messages to patients $[14,15,17,19,20]$. Three (30\%) studies sent reminders to health workers to follow up with patients $[18,21,22]$ and three $(30 \%)$ studies used an mHealth tool to improve patient records or identification [18, $21,22]$. The frequency of these interventions varied widely; educational messages were sent on schedules ranging from daily [19] to twice per month [15]. Some studies specified that appointment reminders were sent a few days in advance of a scheduled appointment $[16,18,23]$ and others did not specify how far in advance patients or health workers were reminded.

Eight $(80 \%)$ of the studies were conducted in Africa [14$16,19-23]$ and two (20\%) were conducted in Asia [17, 18]. All included studies were published between 2010 and 2014, 


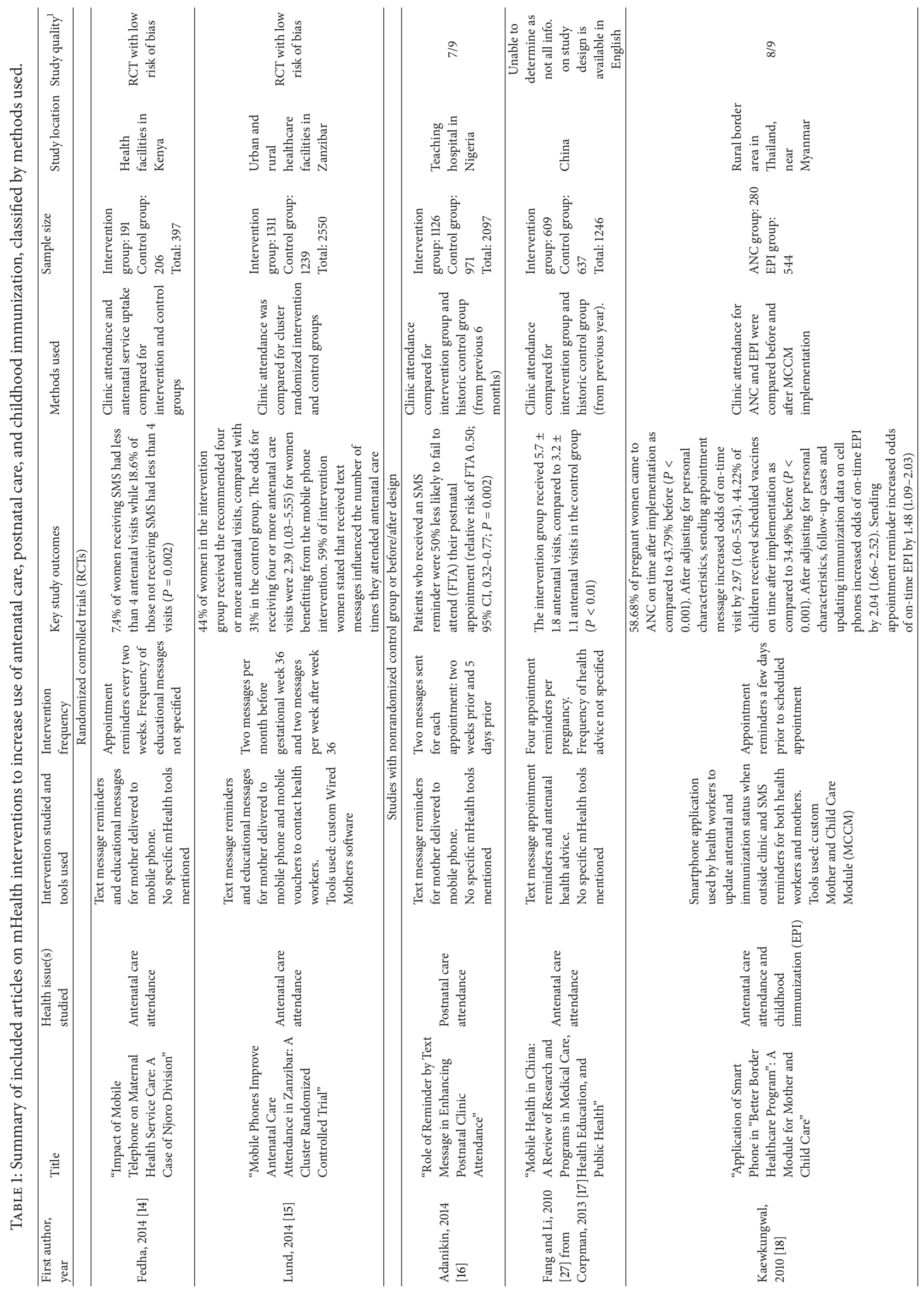




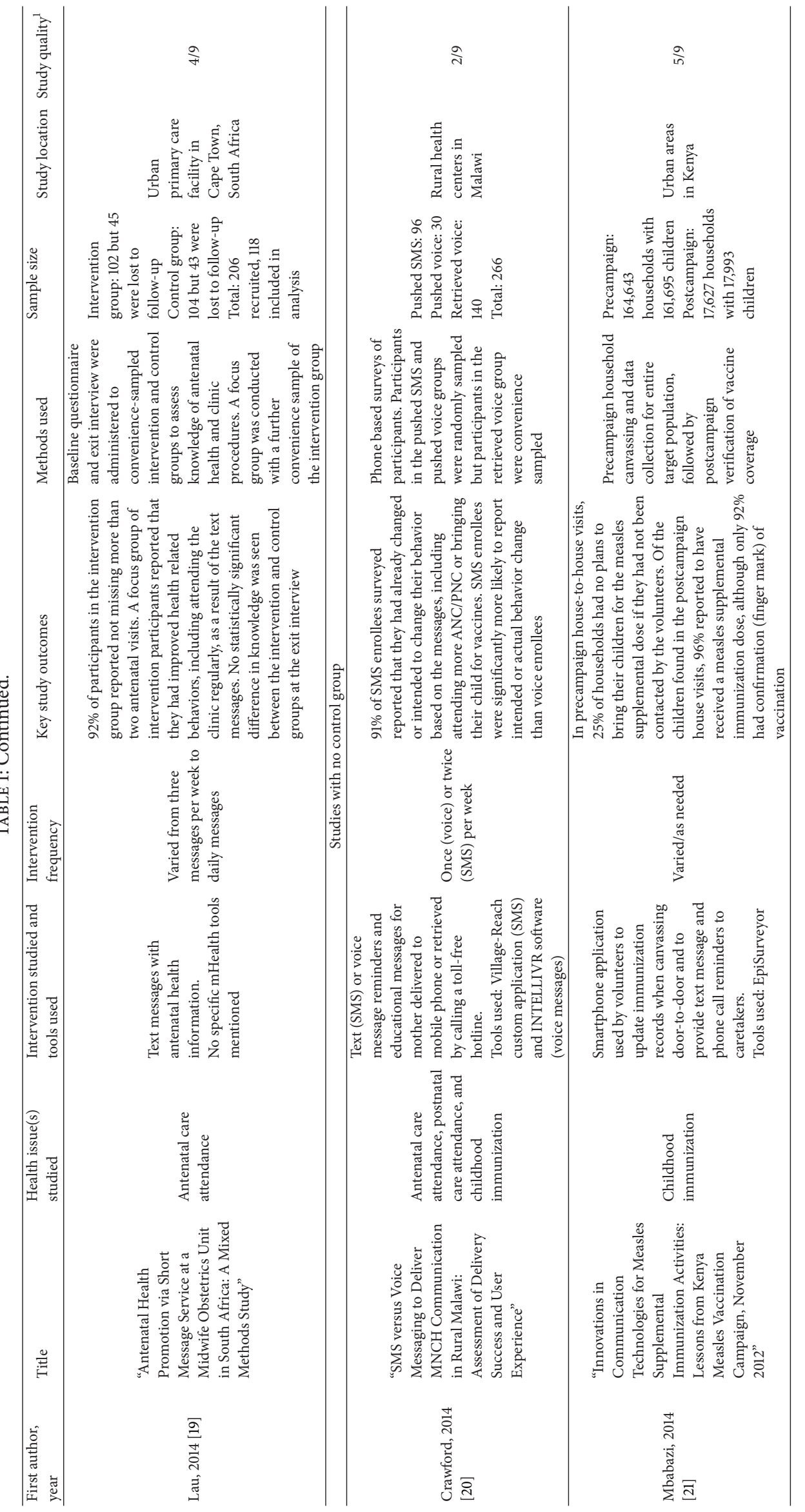




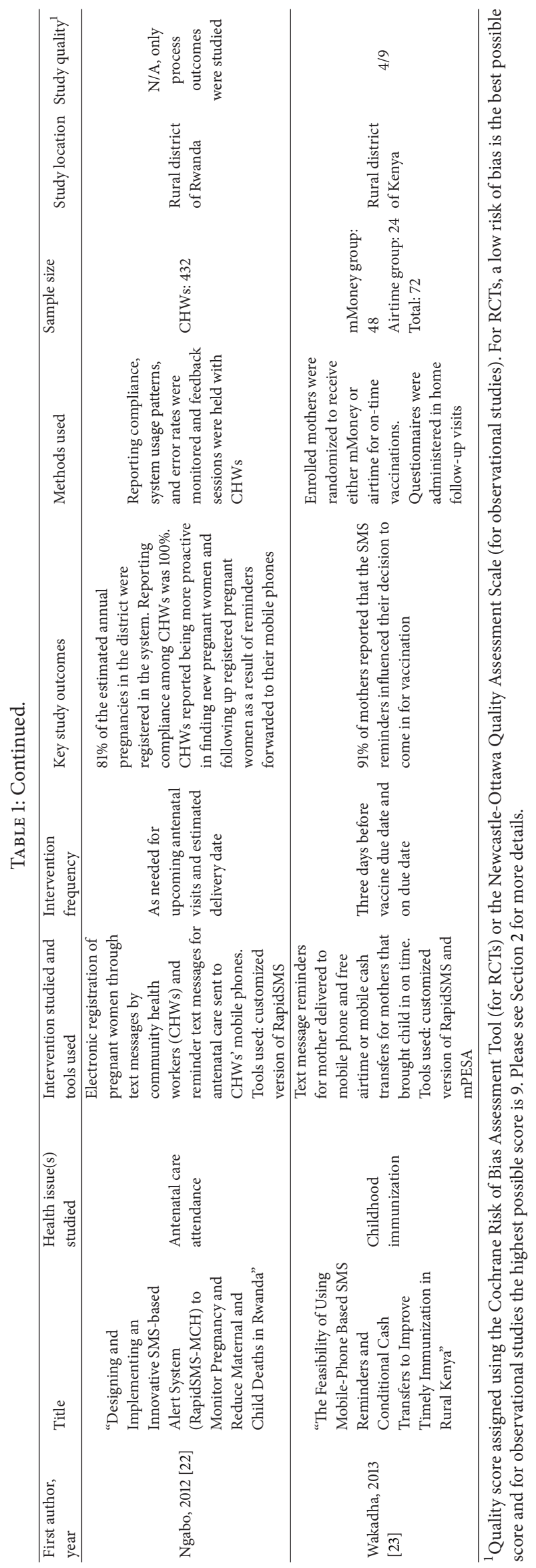


suggesting that the inclusion criterion of studies published after 2000 was sufficiently conservative and that it is unlikely that any relevant articles were missed from earlier publication dates. One study is taken from a literature review published in English on mHealth tools in China [17]; however, the original study was published in Chinese. Therefore, the information available on this study is less complete than that provided for the studies where the primary publication was included.

3.2. Findings by Intervention. All studies showed some evidence that the mHealth intervention implemented had a positive impact on patient or health worker behavior. However, the quality of the studies varied and some of these outcomes cannot be conclusively attributed to the mHealth intervention that was implemented from these studies alone.

3.2.1. Antenatal Care Attendance. Two of the seven studies examining antenatal care attendance were RCTs [14, 15]. Both studies used text message reminders and education for pregnant women and one also provided the women with mobilephone vouchers to contact their health worker, if needed [15]. Both studies found a statistically significant increase of over $10 \%$ in the proportion of women receiving at least four antenatal care visits between the intervention and control groups. Another study examined antenatal care attendance before and after implementation of an mHealth application for improved patient records and automated appointment reminders; this study similarly found a statistically significant improvement in on-time antenatal care attendance following implementation [18]. A study conducted in China sent text message reminders for antenatal care and health advice to an intervention group and found a statistically significant increase in antenatal care attendance, compared to a historic control group. The remaining studies examining antenatal care attendance found some self-reported behavior change from both patients and health workers $[19,20,22]$.

3.2.2. Postnatal Care Attendance. One study examining postnatal care attendance used a historic control group from the previous 6 months in the same hospital and found that the intervention group, receiving text message appointment reminders, were $50 \%$ less likely to fail to attend their appointment $(P=0.002)[16]$. Another study found that women self-reported intended or actual behavior change, including increased attendance to postnatal care, after receiving voice or SMS messages with education and reminders [20].

3.2.3. Childhood Immunization. A study examining childhood immunization found a statistically significant increase (from $34.5 \%$ to $44.2 \%, P<0.001$ ) in the proportion of children receiving on-time vaccination after implementation of a mobile application for improved patient records and automated text message appointment reminders [18]. Another study found that mothers reported being influenced by a text message reminder (which were also tied to a conditional cash transfer, if child was vaccinated on time) to bring their child for immunization [23]. In one study, after receiving SMS or voice reminders and education, mothers self-reported intended or actual behavior change, including bringing their child for vaccines [20]. Finally, a study using an mHealth application to improve records and to send reminders during a mass vaccination campaign found that $92 \%$ of children visited at home following the campaign had received the measles vaccine [21].

3.3. Findings on Cost. Two of the studies included information on the cost of their mHealth interventions. Adanikin et al. reported a total cost of only US $\$ 21.12$ to send 2252 SMS reminders for postnatal care during the six-month study in Nigeria [16]. Ngabo et al. cited initial investment cost as being considerable, largely due to the fact that they provided all community health workers in Rwanda with a mobile phone to "boost engagement and motivation of CHWs." However, ongoing costs were lowered by Ministry of Health negotiations with the private sector, reducing SMS costs from US $\$ 0.05$ to US $\$ 0.005$ per message [22].

\section{Discussion}

Though all included studies showed some evidence that mHealth tools can be effective in changing patient and health worker behavior to increase antenatal care attendance, postnatal care attendance, and childhood immunization rates, the quality of the evidence varied widely.

The strongest evidence exists for text message reminders and education delivered to pregnant women's mobile phones. The two RCTs that examined this intervention both found evidence of statistically significant increases in antenatal care attendance in their intervention groups, relative to their control groups $[14,15]$. There is also some suggestion from the results that this intervention may also be effective when applied to the other health issues studied, such as postnatal care attendance and childhood immunization. Though no RCTs studied these health issues, two observational studies of high quality found evidence of effectiveness. Adanikin et al. found that intervention group receiving text message reminders for postnatal care were $50 \%$ less likely to fail to attend their appointments than a historic control group from the previous six months $(P=0.002)[16]$. Kaewkungwal et al. found that after implementation of a smartphone application that supported record keeping and generated text message reminders for health workers and mothers, there was a $15 \%$ increase in women attending ANC on time $(P<0.001)$ and a $10 \%$ increase in children receiving on-time immunizations $(P<0.001)$ [18].

Beyond these findings, much of the evidence is based on self-reported behavior change from health workers and patients, which is not sufficiently reliable to draw any strong conclusions on the effectiveness of mHealth interventions $[20,22,23]$. Some other observational studies demonstrated good results of their programs, such as $92 \%$ confirmed coverage in a measles vaccine campaign [21]; however, it is impossible to determine which factors influenced the campaign's success and whether it was due to the use of an mHealth intervention or one of the other program components. In addition, several studies combined multiple 
mHealth interventions (e.g., text message reminders and conditional cash transfers via mobile phone [23]), making it impossible to determine to what degree each intervention influenced the resulting behavior change.

As a result of these methodological limitations and the small number of studies meeting the inclusion criteria, further randomized controlled trials are needed to evaluate the effectiveness of mHealth tools for antenatal care, postnatal care, and childhood immunizations. By employing a multiarm or factorial design, researchers may be able to better ascertain which components of mHealth interventions are most effective.

It is also worth noting that many of the mHealth tools studied focused on a single period of time on the maternal, neonatal, and child health $(\mathrm{MNCH})$ continuum. For example, one study focused only on postnatal care, while five others focused only on antenatal care. Given the importance of continued follow-up of families during pregnancy, delivery, postnatal periods, and early childhood, it would be advisable for future mHealth interventions to consider expanding their tools to include more key events along the MNCH continuum [23]. Finally, the majority of these studies were conducted in Africa, suggesting that there is a need for future study of mHealth tools in broader contexts, including Asia and the Pacific, Central and South America, the Caribbean, and other regions.

This literature review has provided us with the key knowledge that there is some existing evidence of the effectiveness of text message reminders for antenatal care, postnatal care, and immunizations and it has also helped to identify that this is an area where further research is needed. Given the limited, but largely positive, results of this literature review, researchers and public health practitioners should continue to implement mHealth tools for antenatal care attendance, postnatal care attendance, and childhood immunization. However, careful evaluation and further research are still needed to better determine how effective these tools are and in which settings.

\section{Conclusions}

Based on a systematic review of the literature, there is some evidence that mHealth tools may present an opportunity to influence behavior change and ensure that women and children in low-income countries are accessing prevention services, including antenatal care, postnatal care, and immunizations. Though mHealth programs have been implemented in low- and middle-income countries all over the world [24], there are few peer-reviewed studies and the majority of evaluations relating to maternal and child health have been conducted in Africa. Therefore, greater emphasis needs to be put on the evaluating mHealth tools and disseminating results to inform program design and policy making. In addition, many existing interventions focus on only one component of maternal and child health preventive services, rather than on design of an integrated system that follows women and children through the maternal, neonatal, and child health continuum [25]. The field of mHealth should continue to be supported and studied as it shows promise of improving the lives of women and children in low- and middle-income countries.

\section{Conflict of Interests}

The authors declare that there is no conflict of interests regarding the publication of this paper.

\section{References}

[1] World Health Organization, Trends in Maternal Mortality: 1990 to 2013, WHO, 2014, http://www.who.int/reproductivehealth/ publications/monitoring/maternal-mortality-2013/en/.

[2] World Health Organization (WHO), Antenatal Care: Situation, World Health Organization, Geneva, Switzerland, 2012, http:// www.who.int/gho/maternal_health/reproductive_health/antenatal_care_text/en/.

[3] C. Warren, P. Daly, L. Toure, and P. Mongi, "Postnatal care," in Opportunities for Africa's Newborns, J. Lawn and K. Kerber, Eds., Partnership for Maternal, Newborn, and Child Health, Save the Children, UNFPA, UNICEF, USAID,WHO, and partners, Cape Town, South Africa, 2006.

[4] UNICEF, Immunization, 2014, http://www.unicef.org/immunization/index_2819.html.

[5] World Health Organization (WHO), Global Health Observatory: Immunization, WHO, Geneva, Switzerland, 2008, http://www.who.int/gho/immunization/en/.

[6] World Health Organization (WHO), mHealth: New Horizons for Health through Mobile Technologies: Second Global Survey on eHealth, WHO, Geneva, Switzerland, 2011, http://www.who.int/ goe/publications/goe_mhealth_web.pdf.

[7] K. A. Kannisto, M. H. Koivunen, and M. A. Välimäki, "Use of mobile phone text message reminders in health care services: a narrative literature review," Journal of Medical Internet Research, vol. 16, no. 10, article e222, 2014.

[8] I. Asangansi and K. Braa, "The emergence of mobile-supported national health information systems in developing countries," in MEDINFO 2010, C. Safran, S. Reti, and H. F. Marin, Eds., IOS Press, 2010.

[9] A. Chib, M. O. Lwin, J. Ang, H. Lin, and F. Santoso, "Midwives and mobiles: using ICTs to improve healthcare in Aceh Besar, Indonesia," Asian Journal of Communication, vol. 18, no. 4, pp. 348-364, 2008.

[10] World Bank, "Data: Countries and Economies," November 2014, http://data.worldbank.org/country.

[11] L. Chen, W. Wang, X. Du et al., "Effectiveness of a smart phone app on improving immunization of children in rural Sichuan Province, China: Study protocol for a paired cluster randomized controlled trial," BMC Public Health, vol. 14, no. 1, article 262, 2014.

[12] J. P. T. Higgins and S. Green, Eds., Cochrane Handbook for Systematic Reviews of Interventions Version 5.1.0, The Cochrane Collaboration, 2011, http://www.cochrane-handbook.org/.

[13] G. A. Wells, B. Shea, D. O'Connell et al., "The Newcastle-Ottawa Scale (NOS) for assessing the quality of nonrandomised studies in meta-analyses," November 2014, http://www.ohri.ca/programs/clinical_epidemiology/oxford.htm.

[14] T. Fedha, "Impact of mobile telephone on maternal health service care: a case of Njoro division," Open Journal of Preventive Medicine, vol. 4, no. 5, pp. 365-376, 2014. 
[15] S. Lund, B. B. Nielsen, M. Hemed et al., "Mobile phones improve antenatal care attendance in Zanzibar: a cluster randomized controlled trial," BMC Pregnancy and Childbirth, vol. 14, no. 1, article 29, 2014.

[16] A. I. Adanikin, J. O. Awoleke, and A. Adeyiolu, "Role of reminder by text message in enhancing postnatal clinic attendance," International Journal of Gynecology \& Obstetrics, vol. 126, no. 2, pp. 179-180, 2014.

[17] D. W. Corpman, "Mobile health in China: a review of research and programs in medical care, health education, and public health," Journal of Health Communication, vol. 18, no. 11, pp. 1345-1367, 2013.

[18] J. Kaewkungwal, P. Singhasivanon, A. Khamsiriwatchara, S. Sawang, P. Meankaew, and A. Wechsart, "Application of smart phone in 'Better Border Healthcare Program': a module for mother and child care," BMC Medical Informatics and Decision Making, vol. 10, article 69, 2010.

[19] Y. K. Lau, T. Cassidy, D. Hacking, K. Brittain, H. J. Haricharan, and M. Heap, "Antenatal health promotion via short message service at a Midwife Obstetrics Unit in South Africa: a mixed methods study," BMC Pregnancy and Childbirth, vol. 14, no. 1, article 284, 2014.

[20] J. Crawford, E. Larsen-Cooper, Z. Jezman, S. C. Cunningham, and E. Bancroft, "SMS versus voice messaging to deliver $\mathrm{MNCH}$ communication in rural Malawi: assessment of delivery success and user experience," Global Health: Science and Practice, vol. 2, no. 1, pp. 35-46, 2014.

[21] W. B. Mbabazi, C. W. Tabu, C. Chemirmir et al., "Innovations in communication technologies for measles supplemental immunization activities: lessons from Kenya measles vaccination campaign, November 2012," Health Policy and Planning, 2014.

[22] F. Ngabo, J. Nguimfack, F. Nwaigwe et al., "Designing and Implementing an Innovative SMS-based alert system (RapidSMS-MCH) to monitor pregnancy and reduce maternal and child deaths in Rwanda," The Pan African Medical Journal, vol. 13, p. 31, 2012.

[23] H. Wakadha, S. Chandir, E. V. Were et al., "The feasibility of using mobile-phone based SMS reminders and conditional cash transfers to improve timely immunization in rural Kenya," Vaccine, vol. 31, no. 6, pp. 987-993, 2013.

[24] World Health Organization (WHO), eHealth and Innovation in Women's and Children's Health: A Baseline Review: Based on the Findings of the 2013 Survey of Coia Countries by the WHO Global Observatory for eHealth, March 2014, World Health Organization, Geneva, Switzerland, 2014, http://www.who.int/goe/publications/en/.

[25] T. Tamrat and S. Kachnowski, "Special delivery: an analysis of mhealth in maternal and newborn health programs and their outcomes around the world," Maternal and Child Health Journal, vol. 16, no. 5, pp. 1092-1101, 2012.

[26] D. Moher, A. Liberati, J. Tetzlaff, D. G. Altman, and The PRISMA Group, "Preferred reporting items for systematic reviews and meta-analyses: the PRISMA statement," PLOS Medicine, vol. 6, no. 7, Article ID e1000097, 2009.

[27] W. J. Fang and J. J. Li, "Application of SMS to perinatal health service for migrant pregnant women," Journal of Nursing, vol. 17, no. 8, pp. 42-44, 2010. 


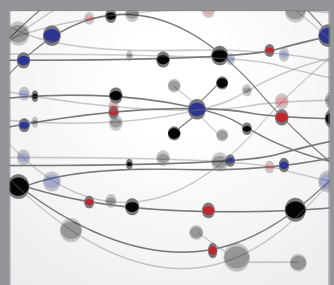

The Scientific World Journal
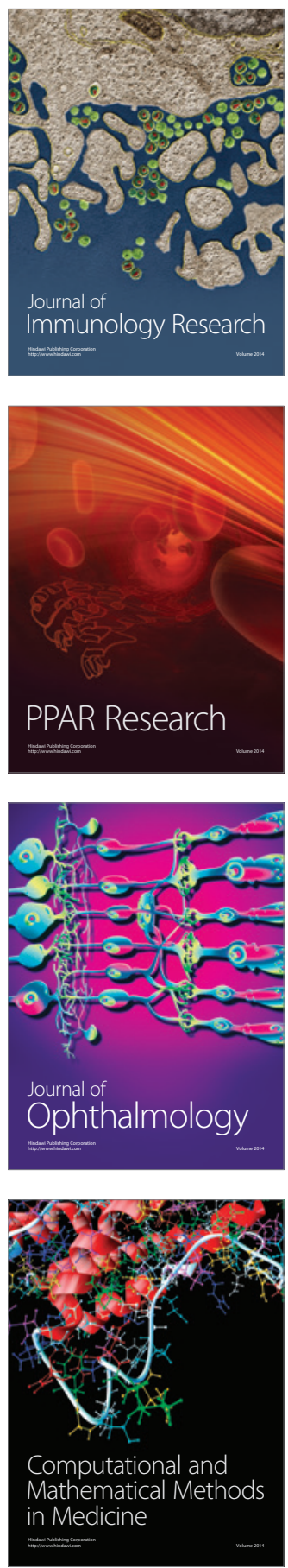

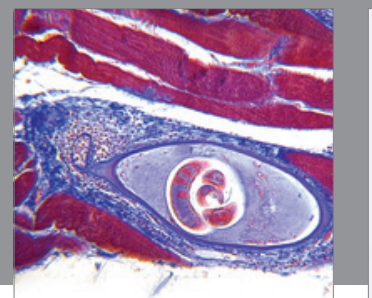

Gastroenterology

Research and Practice
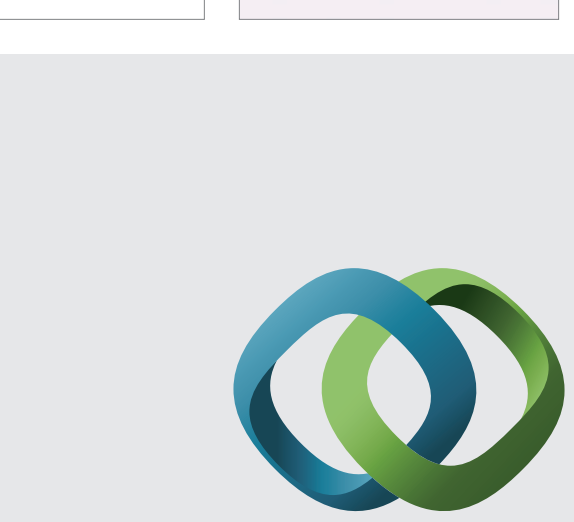

\section{Hindawi}

Submit your manuscripts at

http://www.hindawi.com
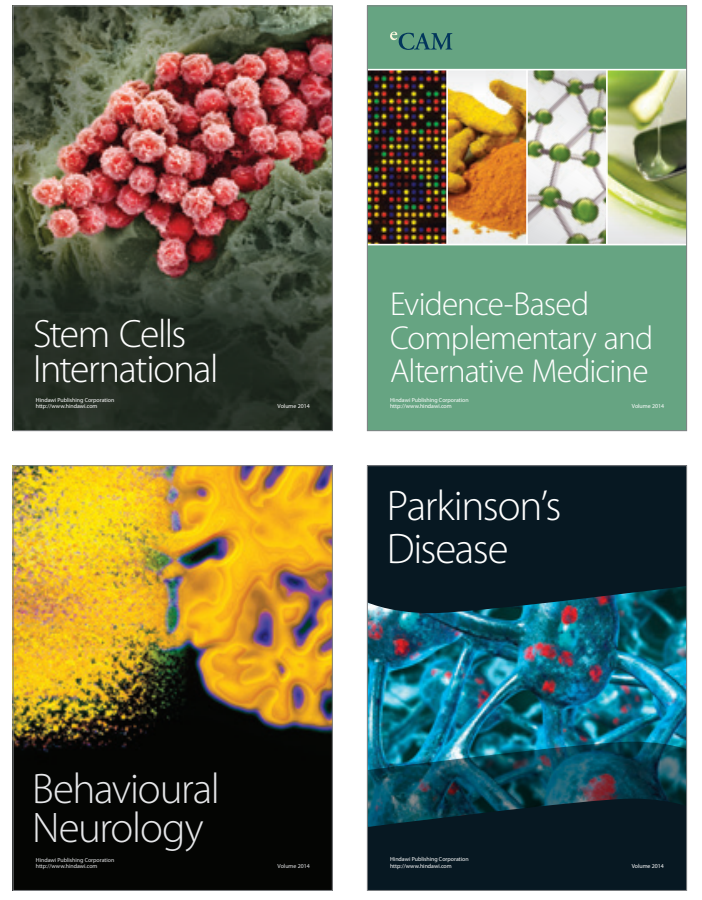
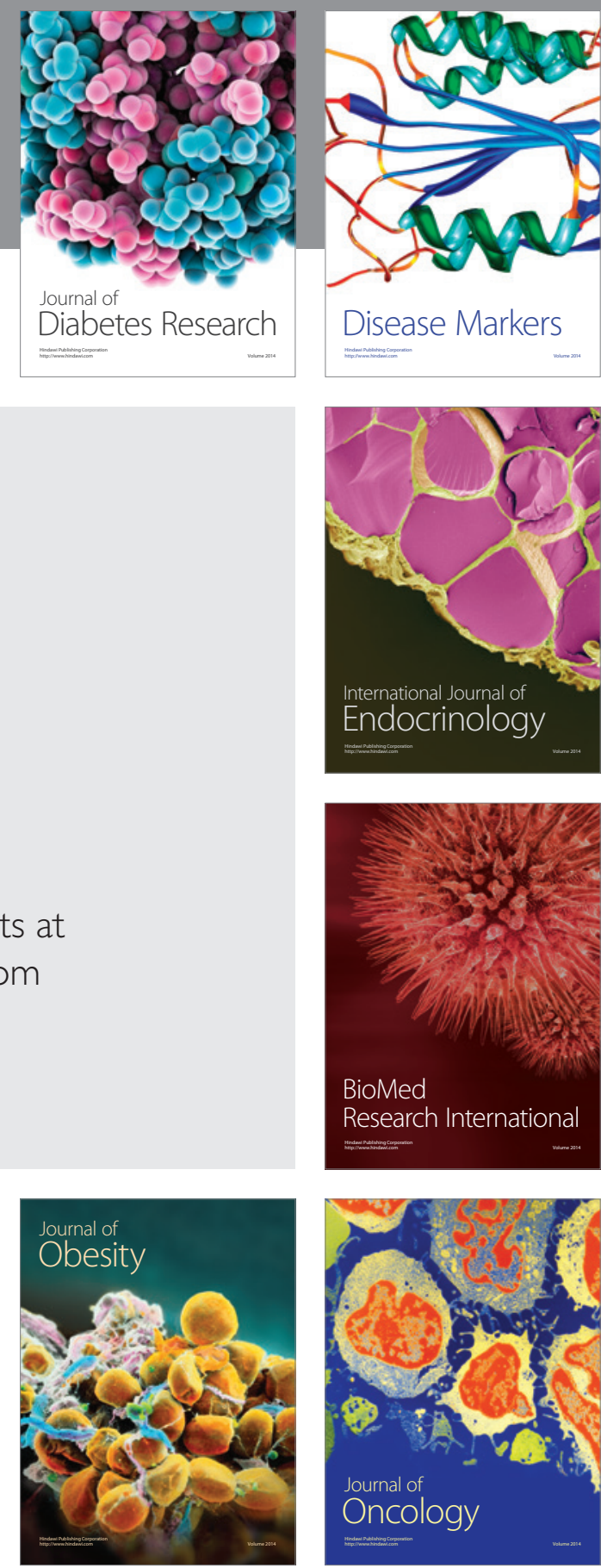

Disease Markers
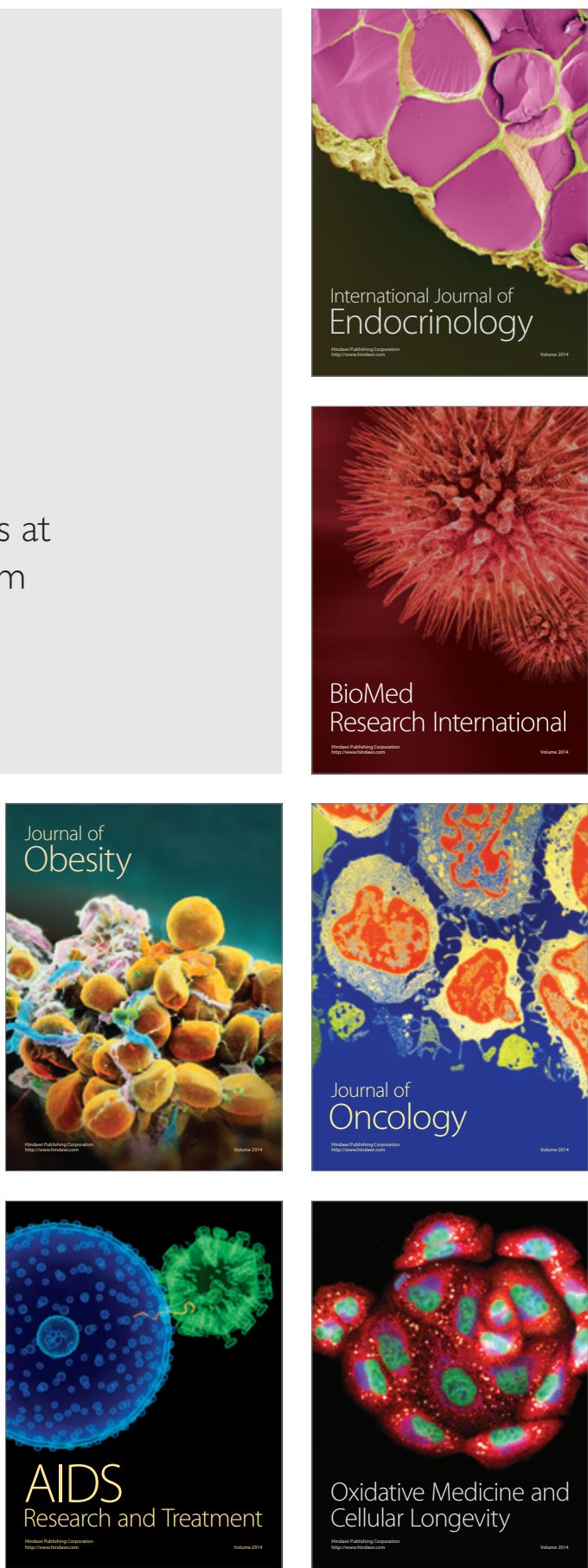